\title{
SUSCEPTIBILITY OF THE HOUSE FLY, MUSCA DOMESTICA (DIPTERA: MUSCIDAE), TO THE ENTOMOGENOUS NEMATODES STEINERNEMA FELTIAE, S. GLASERI (STEINERNEMATIDAE), AND HETERORHABDITIS HELIOTHIDIS (HETERORHABDITIDAE) ${ }^{1}$
}

\author{
C.J. Geden, R.C. Axtell, and W.M. Brooks ${ }^{2}$
}

\begin{abstract}
The infectivity of the nematodes Steinernema feltiae, S. glaseri, and Heterorhabditis heliothidis for the larval, pupal, and adult stages of the house fly were evaluated under different habitat conditions. Second- and 3rd-instar larvae and adult flies were highly susceptible to $S$. feltiae and $H$. heliothidis when hosts were confined in petri dishes containing nematode-treated filter paper. Larvae were not susceptible to $S$. glaseri, and pupae within puparia were refractory to infection by all 3 species. When 2nd- and 3rd-instar larvae were exposed to nematodes in rearing medium, S. feltiae caused higher mortality (55-61\%) than $H$. heliothidis (11-26\%) at the highest dosage of 5,000 nematodes per host. Both $S$. feltiae and $H$. heliothidis were more infective for 3rd-instar larvae (21-29\%) than for 2nd-instar larvae (2$6 \%)$ at this dosage in poultry manure. When adult flies were offered S. feltiae suspensions in a $5 \%$ sucrose bait on cotton balls, mortality ranged from 53 to $67 \%$ at dose rates ranging from 1,000 to 100,000 nematodes per milliliter of bait.
\end{abstract}

Nematodes of the genera Steinernema $[=\mathrm{NeO}$ aplectana (Wouts et al. 1982)] (Steinernematidae) and Heterorhabditis (Heterorhabditidae) parasitize a wide range of insect hosts (Poinar 1975, 1979). Their infectivity for insects, persistence, and lack of pathogenicity to mammals and birds (Poinar et al. 1982) render these nematodes promising biocontrol agents against various pests.

Most research with entomogenous nematodes has been concerned with their infectivity for lepidopteran and coleopteran pests of agricultural importance. Relatively few Diptera have been studied. Steinernema feltiae (Filipjev) $(=N$. carpocapsae Weiser) parasitizes species of Culicidae (Welch \& Bronskill 1962; Laumond et al. 1979), Simuliidae (Webster 1973), Cecidomyiidae (Laumond et al. 1979), Tipulidae (Lam \& Webster 1972), Tephritidae (Beavers \& Calkins 1984), Anthomyiidae (Welch \& Briand 1961; Cheng \& Bucher 1972), Tachinidae (Kaya 1984), Glossinidae (Poinar et al. 1977), and Calliphoridae (Bedding et al. 1983). Other entomogenous nematodes, including S. glaseri (Steiner)

1. Paper No. 9926 of the Journal Series of the North Carolina Agricultural Research Service, Raleigh, North Carolina 27695-7601, USA.

2. Department of Entomology, North Carolina State University, Raleigh, North Carolina 27695-7613, USA. and Heterorhabditis heliothidis (Khan, Brooks \& Hirschmann), reportedly infect some tephritid and calliphorid flies (Bedding et al. 1983; Beavers \& Calkins 1984).

There is little information on the infectivity of these nematodes for the house fly, Musca domestica L., even though this fly is a major economic pest and an object of biocontrol efforts (Axtell 1970, 1981; Patterson et al. 1981). Dutky et al. (1956) included house fly larvae and pupae in a list of insects that were susceptible to S. feltiae, and Laumond et al. (1979) reported that adult flies were susceptible to this parasite while larvae and eggs were not. Khan et al. (1976) found that house fly larvae were not susceptible to $H$. heliothidis. Few details were given in these reports about the method of exposure, the substrate, or the criteria for determining susceptibility. We therefore examined the infectivity of the 3 entomogenous nematodes S. feltiae, S. glaseri, and H. heliothidis for various life stages of the house fly in different habitat conditions.

\section{MATERIALS AND METHODS}

Nematode and fly cultures. The DD-136 strain of S. feltiae was used, while cultures of S. glaseri and $H$. heliothidis were established from more recent North Carolina isolates from Japanese beetle larvae and soil, respectively (Khan et al. 1976; Regniere \& Brooks 1978). Nematodes were reared by passage through late-instar larvae of the greater wax moth, Galleria mellonella (L.), using methods slightly modified from those of Dutky et al. (1964). After harvest of the infective juveniles from host cadavers, the nematodes were stored in tissue-culture flasks in incubators at $16{ }^{\circ} \mathrm{C}$.

House flies were from a 1-year-old colony initially collected from a poultry house in North Carolina. Larvae were reared on a mixture of fly rearing medium (Ralston Purina, St. Louis, MO), yeast, and water ("CSMA medium"). Adults were provided with milk and granulated sucrose. Under 
these fly rearing conditions $\left(26^{\circ} \mathrm{C}\right)$, 2nd-instar larvae were obtained for experiments by removing larvae from the medium $48 \mathrm{~h}$ after the introduction of fly eggs, and 3rd-instar larvae were obtained by removal after 5 days.

Susceptibility of fly immatures on filter paper. Steinernema feltiae and $H$. heliothidis were tested $\left(26^{\circ} \mathrm{C}\right)$ against house fly larvae by placing 10 larvae in a petri dish lined with a 9-cm-diam disk of filter paper and adding $1 \mathrm{ml}$ of a nematode suspension in water at dosage levels of $0,1,10,100,1,000$, and 5,000 nematodes per larva. In tests with 2 nd-instar larvae, mortality was recorded 3 days following exposure to parasites. The same protocol was followed for 3rd-instar larvae except that mortality was based on adult fly emergence. Cadavers from each dosage group were subsequently held and examined for nematode reproduction and exit.

Tests with $S$. glaseri were conducted using the same procedure after making adjustments for the larger size of the infective juveniles of this species; 2nd-instar larval tests were omitted, and the maximum dosage tested against 3rd-instar larvae was 1,000 nematodes per host.

The ability of the 3 nematode species to penetrate fly puparia was assessed by placing ten 2 -dayold pupae (within puparia) on filter paper in a petri dish $\left(26^{\circ} \mathrm{C}\right)$ and exposing them to a single dosage of 10,000 nematodes or water-only controls (10 dishes/species). The number of adult flies that emerged was counted 6 days posttreatment. Puparia from which flies failed to emerge were dissected, and the pupae were examined for the presence of nematodes.

Susceptibility of fly larvae in rearing medium. When the results of the above tests indicated that $S$. glaseri had low infectivity for larvae, only $S$. feltiae and $H$. heliothidis were used in subsequent tests. To examine the susceptibility of fly larvae to these nematodes in a seminatural substrate, 2nd- or 3rd-instar larvae were placed in $150 \mathrm{~cm}^{3}$ of larval medium in screen-topped plastic cups (depth of medium $=5$ $\mathrm{cm}$ ) and treated with $1 \mathrm{ml}$ of nematode suspensions at the same dosage rates used in the tests on filter paper. Five cups containing 20 larvae were tested for each dosage, nematode species, and host larval stage. Mortality was based on adult fly emergence at 2 wk posttreatment.

Susceptibility of fly larvae in poultry manure. Steinernema feltiae and $H$. heliothidis were tested against house fly larvae in poultry manure collected from beneath the cages of white leghorn laying hens. Manure (ca. $72 \%$ moisture) was frozen and thawed before use to kill extraneous arthropods. Three groups of tests were conducted in this series. In the initial test, 2 nd- or 3 rd-instar larvae were added to $150 \mathrm{~cm}^{3}$ of manure in a plastic cup and treated with 1 milliliter of nematode suspensions at the same dosage rates used in the tests with filter paper and rearing medium. Five cups containing 20 larvae/cup were tested for each dosage, nematode species, and host larval stage.

In the 2nd group, cups with manure as before were inoculated with 50 fresh fly eggs per cup and treated with nematodes either 2 days (2nd-instar larvae) or 5 days (3rd-instar larvae) later to allow larvae to condition the manure before nematode application. Dosage levels of 0, 1,000, 10,000, and 100,000 nematodes/cup were used. Five cups with 50 eggs/cup were used for each dosage, nematode species, and fly larval stage. In the 3rd group, high larval density conditions were first established by adding $5 \mathrm{~cm}^{3}$ of fly eggs to 12 liters of manure in a large pan $(34.5 \times 47.0 \times 12.0 \mathrm{~cm})$. After 5 days the pan containing manure and larvae was chilled to $10^{\circ} \mathrm{C}$ to minimize larval movement. Manure and larvae were gently mixed to randomize larval distribution in the manure. The manure was then divided into 80 subsamples of equal size $\left(150 \mathrm{~cm}^{3}\right.$ of manure plus larvae), added to screen-topped plastic cups, and held at $26^{\circ} \mathrm{C}$. Three hours later, nematodes were introduced into the manure at dosage levels of $0,1,000,10,000$, and 100,000 per cup (10 cups/dose/species). Mortality was based on adult fly emergence 2 wk posttreatment.

Susceptibility of adult flies to nematodes. In 1 test group, 4-day-old flies were exposed to nematodetreated filter paper in petri dishes using the same procedures used in tests with larvae, except that flies were provided with cubes of granulated sucrose throughout the test. Steinernema feltiae, $S$. glaseri, and $H$. heliothidis were all evaluated at the same dosage levels used in the larval tests.

Flies were also exposed to sucrose solutions containing infective juveniles of $S$. feltiae and $H$. heliothidis. As nematode survival and infectivity were not adversely affected by solutions containing up to $10 \%$ sucrose in preliminary tests, 2 delivery systems for fly feeding were evaluated, using nematodes in a $5 \%$ sucrose bait. In the first, 4-day-old flies were placed in plastic drinking cups and provided with pieces of absorbant cotton moistened to saturation with $4 \mathrm{ml}$ of a $5 \%$ sucrose solution 
TABLE 1. Infectivity of Steinernema feltiae, S. glaseri, and Heterorhabditis heliothidis against Musca domestica larvae and adults on nematode-treated filter paper, and of $S$. feltiae and $H$. heliothidis against fly larvae in rearing medium and poultry manure.

\begin{tabular}{|c|c|c|c|c|c|}
\hline \multirow[b]{2}{*}{ FLY STAGE } & \multicolumn{5}{|c|}{ MEAN \% MORTALITY* AT NEMATOdE DOSE (NO./HOST) } \\
\hline & 1 & 10 & 100 & 1,000 & 5,000 \\
\hline \multicolumn{6}{|l|}{ Filter paper substrate } \\
\hline \multicolumn{6}{|l|}{ S. feltiae } \\
\hline Larvae, 2nd instar & $15.6 \mathrm{a}^{* *}$ & $18.9 \mathrm{~b}$ & $44.4 \mathrm{~b}$ & $76.6 \mathrm{c}$ & $100 \mathrm{a}$ \\
\hline Larvae, 3rd instar & $0 \mathrm{~b}$ & $15.6 \mathrm{~b}$ & $39.6 \mathrm{~b}$ & $56.2 \mathrm{~cd}$ & $92.7 \mathrm{~b}$ \\
\hline Adults & $0 \mathrm{~b}$ & $15.2 \mathrm{~b}$ & $26.0 \mathrm{c}$ & $86.4 \mathrm{~b}$ & $100 \mathrm{a}$ \\
\hline \multicolumn{6}{|l|}{ H. heliothidis } \\
\hline Larvae, 2nd instar & $18.4 \mathrm{a}$ & $26.4 \mathrm{a}$ & $65.5 \mathrm{a}$ & $97.7 \mathrm{a}$ & $100 \mathrm{a}$ \\
\hline Larvae, 3rd instar & $0 \mathrm{~b}$ & $1.1 \mathrm{c}$ & $27.6 \mathrm{c}$ & $69.1 \mathrm{c}$ & $97.9 \mathrm{ab}$ \\
\hline Adults & $0 \mathrm{~b}$ & $10.1 \mathrm{~b}$ & $34.8 \mathrm{bc}$ & $73.0 \mathrm{c}$ & $91.0 \mathrm{~b}$ \\
\hline \multicolumn{6}{|l|}{ S. glaseri } \\
\hline Larvae, 3rd instar & $0 \mathrm{~b}$ & $1.1 \mathrm{c}$ & $1.1 \mathrm{e}$ & $0 \mathrm{e}$ & - \\
\hline Adults & $2.2 \mathrm{~b}$ & $2.2 \mathrm{c}$ & $11.8 \mathrm{~d}$ & $49.5 \mathrm{~d}$ & - \\
\hline \multicolumn{6}{|c|}{ Rearing medium substrate } \\
\hline \multicolumn{6}{|c|}{ S. feltiae } \\
\hline Larvae, 2nd instar & $0 \mathrm{a}$ & $0 \mathrm{a}$ & $3.3 \mathrm{c}$ & $47.2 \mathrm{a}$ & $60.4 \mathrm{a}$ \\
\hline Larvae, 3rd instar & $0 \mathrm{a}$ & $3.3 \mathrm{a}$ & $20.0 \mathrm{a}$ & $27.8 \mathrm{~b}$ & $55.6 \mathrm{a}$ \\
\hline \multicolumn{6}{|l|}{ H. heliothidis } \\
\hline Larvae, 2nd instar & $1.1 \mathrm{a}$ & $0 \mathrm{a}$ & $7.7 \mathrm{~b}$ & $3.3 \mathrm{c}$ & $11.0 \mathrm{C}$ \\
\hline Larvae, 3rd instar & $1.1 \mathrm{a}$ & $0 \mathrm{a}$ & $2.2 \mathrm{c}$ & $0 \mathrm{c}$ & $25.6 \mathrm{~b}$ \\
\hline \multicolumn{6}{|c|}{ Poultry manure substrate } \\
\hline \multicolumn{6}{|l|}{ S. feltiae } \\
\hline Larvae, 2nd instar & $2.4 \mathrm{a}$ & $2.4 \mathrm{a}$ & $4.8 \mathrm{a}$ & $3.6 \mathrm{a}$ & $2.4 \mathrm{~b}$ \\
\hline Larvae, 3rd instar & $2.4 \mathrm{a}$ & $0 \mathrm{a}$ & $0 \mathrm{a}$ & $0 \mathrm{a}$ & $28.2 \mathrm{a}$ \\
\hline \multicolumn{6}{|l|}{ H. heliothidis } \\
\hline Larvae, 2nd instar & $2.4 \mathrm{a}$ & $7.1 \mathrm{a}$ & $10.7 \mathrm{a}$ & $1.2 \mathrm{a}$ & $5.9 \mathrm{~b}$ \\
\hline Larvae, 3 rd instar & $1.1 \mathrm{a}$ & $6.8 \mathrm{a}$ & $4.5 \mathrm{a}$ & $0 \mathrm{a}$ & $21.6 \mathrm{a}$ \\
\hline
\end{tabular}

* Corrected for control mortality by Abbott's formula.

** Means within columns (dose rates) under each subheading followed by the same letter are not significantly different $(P<$ 0.05, Duncan's multiple range test).

containing $0,1,000,10,000$, or 100,000 nematodes per milliliter. The cotton was remoistened with water twice daily for 3 days, and fly mortality was monitored daily. Five cups with 20 flies per cup were tested at each dosage for both nematode species. A 2nd series of tests was conducted using the same procedure, except that $15-\mathrm{cm}$ cubes of conventional cellulose sponge (O-Cel-O, General Mills, Inc., Tonawanda, NY) were used instead of cotton.

Data analysis. Mean percent mortalities at each nematode dosage rate for tests conducted in the same substrate (filter paper, rearing medium, manure) were analyzed by 1-way analysis of variance and Duncan's multiple range test. Separate analyses were performed on manure test data where host larval densities were set at higher levels and in tests with adult flies fed on nematode-sucrose suspensions. After correcting for control mortality (Abbott 1925), $\mathrm{LD}_{50}$ and $\mathrm{LD}_{90}$ values were computed for filter paper and rearing medium tests by using the log-probit procedure for the Statistical Analysis System (Ray 1982).

\section{RESULTS}

Susceptibility of fly larvae on filter paper. Results of all larval tests (except for manure tests at high larval densities) are presented in Table 1. Mean control mortality ranged from $10-13 \%$ and $4-9 \%$ in tests with 2nd- and 3rd-instar larvae, respectively. Both S. feltiae and H. heliothidis had high infectivity for 2nd-instar fly larvae, with the latter species showing higher virulence than $S$. feltiae under the artificial conditions presented by filter paper. $\mathrm{LD}_{50}$ values were 75 and 23 nematodes/host for $S$. feltiae and $H$. heliothidis, respectively, with corresponding $\mathrm{LD}_{90}$ values of 3,581 (slope $=0.90$ ) 
and 548 (slope $=1.02$ ) parasites per host larva. Third-instar larvae were less susceptible than 2nd instars, with $\mathrm{LD}_{50}$ 's of 262 and 323 for S. feltiae and $H$. heliothidis, respectively. $\mathrm{LD}_{90}$ values were $8,222$ (slope $=0.86)$ for $S$. feltiae and 2,613 (slope $=$ 1.41) for $H$. heliothidis. No nematode-induced mortality was observed with $S$. glaseri, even at the highest dosage of 1,000 parasites per host.

Inspection of parasitized larval cadavers over several weeks following infection revealed that $S$. feltiae successfully reproduced and produced new infective juveniles at all dosage levels tested. No new juveniles were observed in cadavers parasitized by $H$. heliothidis at any dosage level, although such larvae became reddish, a characteristic of infection with this nematode and its associated bacterium (Khan et al. 1976).

Exposure of fly pupae to large dosages $(10,000$ nematodes/host) of all 3 nematodes resulted in no significant mortality, although subsequent dissection of pupae that failed to eclose indicated that nematodes had entered and parasitized a small number of pupae. One percent of the pupae exposed to $S$. feltiae and $2 \%$ of those exposed to $H$. heliothidis contained live nematodes. No nematodes were found in $S$. glaseri-exposed or control pupae.

Susceptibility of fly larvae in rearing medium. Mortality was less than $26 \%$ among 2 nd- and 3rd-instar larvae treated with $H$. heliothidis (Table 1). Steinernema feltiae in this substrate was more infective for 2nd-instar larvae $\left(\mathrm{LD}_{50}=2,049, \mathrm{LD}_{90}=25,305\right.$, slope $=1.17)$ than for 3rd-instar larvae $\left(\mathrm{LD}_{50}=\right.$ $4,036, \mathrm{LD}_{90}=389,416$, slope $\left.=0.64\right)$. Mean control mortalities were 9 and $10 \%$ among 2nd- and 3rd-instar larvae, respectively.

Susceptibility of fly larvae in poultry manure. Nematodes caused low mortality of 2nd-instar fly larvae $(<11 \%)$ in poultry manure (20 fly larvae/cup) at all dosages (Table 1). Some mortality was observed among 3rd-instar larvae at the highest dosage of 5,000 nematodes per larva, with corrected mortalities of 28.2 and $21.6 \%$ among larvae exposed to $S$. feltiae and $H$. heliothidis, respectively. Mean control mortalities ranged from 12 to $16 \%$ among 2nd- and 3rd-instar larvae, respectively.

When host density was increased from 20 larvae per container to initial levels of 50 eggs per container, no significant effect on emergence was noted among larvae treated as 2 nd instars ( 2 days following introduction of fly eggs) (Table 2). When 3rd-instar larvae were treated, however, both
TABLE 2. Mortality of house fly immatures in poultry manure at an initial density of 50 fly eggs per $150 \mathrm{~cm}^{3}$ of manure after treatment with $S$. feltiae and $H$. heliothidis.

\begin{tabular}{lccc}
\hline & \multicolumn{3}{c}{$\begin{array}{c}\text { MEAN \% MORTAlity* AT } \\
\text { NEMATODE DOSE (NO./50 HOSTS) }\end{array}$} \\
\cline { 2 - 4 } \multicolumn{1}{c}{ Fly STAGe } & $10^{3}$ & $10^{4}$ & $10^{5}$ \\
\hline S. feltiae & & & \\
Larvae, 2nd instar & $0 \mathrm{~b} * *$ & $0 \mathrm{~b}$ & $1.8 \mathrm{c}$ \\
Larvae, 3rd instar & $0 \mathrm{~b}$ & $12.9 \mathrm{~b}$ & $47.1 \mathrm{~b}$ \\
H. heliothidis & & & \\
Larvae, 2nd instar & $0 \mathrm{~b}$ & $6.6 \mathrm{~b}$ & $6.6 \mathrm{c}$ \\
Larvae, 3rd instar & $28.1 \mathrm{a}$ & $62.5 \mathrm{a}$ & $67.2 \mathrm{a}$ \\
\hline
\end{tabular}

* Corrected for control mortality by Abbott's formula.

** Means within columns (dose rates) followed by the same letter are not significantly different $(P<0.05$, Duncan's multiple range test).

nematode species significantly reduced fly emergence.

In the final tests with 3rd-instar larvae under high-density conditions, only S. feltiae at 100,000 nematodes per container of manure had a significant $(P<0.05)$ effect on fly emergence, with a mean of $171.4 \pm 25.0$ flies emerging per container compared with $281.0 \pm 33.4$ flies among the controls (not presented in table). No significant effect on emergence was noted with $H$. heliothidis at any dosage level tested.

Susceptibility of adult flies to nematodes. Steinernema glaseri had low infectivity for flies (Table 1). In contrast, fly adults were highly susceptible to $S$. feltiae and $H$. heliothidis. $\mathrm{LD}_{50}$ and $\mathrm{LD}_{90}$ values for $S$. feltiae were 144 and 1,641 nematodes/host, respectively (slope $=1.22$ ), whereas the corresponding values for $H$. heliothidis were 229 and 4,752 nematodes per host (slope $=0.97$ ). Mean control mortalities ranged from 4 to $11 \%$.

Results of tests with adult flies confined with nematodes contained in a $5 \%$ sucrose bait are presented in Table 3. Mortality ranged from 53 to $67 \%$ when flies fed on S. feltiae suspensions presented in cotton at dosage rates ranging from 1,000 to 100,000 nematodes per milliliter of bait. Mortality was less than $30 \%$ at all dosage rates for $S$. feltiae in cellulose sponges and $H$. heliothidis in cotton. Heterorhabditis heliothidis was twice as effective (44.1\% fly mortality) as S. feltiae $(19.0 \%)$ when nematodes were presented in sponges.

\section{DISCUSSION}

Infectivity of nematodes for fly larvae and pupae. Results of the dosage-mortality tests of fly 
TABLE 3. Mortality of 4-day-old house fly adults following a 3 -day exposure to nematode suspensions in a $5 \%$ sucrose bait. Baits were presented in balls of absorbant cotton or in cubes of cellulose sponge material.

\begin{tabular}{cccc}
\hline & \multicolumn{3}{c}{$\begin{array}{c}\text { Mean \% MORTAlity* AT Nematode } \\
\text { DOSE (NO./Milliliter OF Bait) }\end{array}$} \\
\cline { 2 - 4 } Absorbant & \multicolumn{1}{c}{$10^{4}$} & $10^{5}$ \\
\hline Substrate & $10^{3}$ & & \\
\hline S. feltiae & & & \\
Cotton & $53.3 \mathrm{a} * *$ & $62.7 \mathrm{a}$ & $66.7 \mathrm{a}$ \\
Sponge & $12.6 \mathrm{bc}$ & $10.1 \mathrm{c}$ & $19.0 \mathrm{c}$ \\
H. heliothidis & & & \\
Cotton & $16.9 \mathrm{~b}$ & $27.3 \mathrm{~b}$ & $29.9 \mathrm{bc}$ \\
Sponge & $5.9 \mathrm{c}$ & $19.4 \mathrm{bc}$ & $44.1 \mathrm{~b}$ \\
\hline
\end{tabular}

* Corrected for control mortality (range $=7$ to $25 \%$ ) by Abbott's formula.

** Means within columns (dose rates) followed by the same letter are not significantly different $(P<0.05$, Duncan's multiple range test).

larvae on filter paper fall within the range reported for other muscoid flies. Bedding et al. (1983), who examined the susceptibility of several insects to entomogenous nematodes, reported $\mathrm{LD}_{50}$ values of $774 \mathrm{H}$. heliothidis (North Carolina strain) and 285 S. feltiae (Agriotis strain) per mature larva of Calliphora vicina Robineau-Desvoidy. Third-instar larvae of the sheep strike blow fly, Lucilla cuprina (Wd.), were even more susceptible, with $\mathrm{LD}_{50}$ 's of $295 \mathrm{H}$. heliothidis and $71 \mathrm{~S}$. feltiae.

One aspect of our tests not reflected in the overall mortality and $\mathrm{LD}_{50}$ values was the time required for the nematodes to kill their hosts. In all tests on filter paper, including those with adult flies, S. feltiae infections led to host death much more rapidly than did infections with $H$. heliothidis. This difference was also noted in tests with 3rd-instar larvae, where fly emergence was used to determine mortality rather than failure to pupate. Many larvae became infected, pupated, and then died as pupae. Such transtadial passage of nematodes was more common in larvae infected with $H$. heliothidis than in those with $S$. feltiae infections. For example, at the highest nematode dosage of 5,000 parasites per larva, $68 \%$ of the larvae exposed to S. feltiae died as larvae, compared with $31 \%$ of larvae exposed to $H$. heliothidis at this dosage. When pupal mortality was taken into account as well, over $90 \%$ overall mortality was observed among both exposure groups.

House fly pupae were refractory to parasitism, presumably owing to the formidable barrier presented by the puparium. A few pupae contained nematodes upon dissection; these infections may have been a result of physical damage to the puparia during handling before placement in petri dishes. Similar results with fly pupae were obtained by Kaya (1984) with the tachinid fly Compsilura concinnata (Mg.) and by Beavers \& Calkins (1984) with the Caribbean fruit fly Anastrepha suspensa (Loew).

Because pupae within puparia do not become infected, the lower susceptibility of 3rd-instar larvae compared with 2 nd-instar larvae in our tests was probably due to the shorter effective exposure period of the older larvae, since most pupated within $24 \mathrm{~h}$ of being placed in the dishes. Other factors may have played a role as well. Owing to its mechanism of host entry, Steinernema feltiae is restricted to natural openings, while $H$. heliothidis can also penetrate cuticle (Bedding \& Molyneux 1982). The opportunities for per os infection were reduced for both nematode species, since most of the 3rd-instar larvae used in our tests were no longer feeding at the time of exposure to the nematodes. Kaya (1984) reported a low infection rate $(10 \%)$ among tachinid larvae that entered nematode-treated soil to pupate. Cuticular changes before pupation may further reduce the likelihood of successful infection by $H$. heliothidis.

Dutky et al. (1956) reported that house fly larvae were susceptible to $S$. feltiae, while Laumond et al. (1979) found that they were not, even when larvae were exposed to dosages of 10,000 nematodes. It may be that larvae in the latter study were so close to pupation that an insufficient effective exposure period was provided.

Steinernema glaseri had no effect on 3rd-instar fly larvae. Although Molyneux et al. (1983) found that this parasite was effective against mature larvae of C. vicina and L. cuprina, Beavers \& Calkins (1984) obtained an infection rate of only $15.7 \%$ among $A$. suspensa larvae exposed to 1,000 S. glaseri. The large size of infective juveniles of this species may render it an unsuitable parasite against smallerbodied hosts with smaller natural entry points.

Although fly larvae were highly susceptible to $S$. feltiae and $H$. heliothidis on moist filter paper, neither nematode species was very infective for fly larvae in rearing medium or poultry manure. At high dosages $S$. feltiae did limit fly emergence by $55-60 \%$ when applied to 2 nd- and 3rd-instar larvae in fly rearing medium. Heterorhabditis heliothidis was less infective at all dosages in this substrate. This difference may have been due in part to different host-seeking behaviors of the nematodes. The infective-stage juveniles of $S$. feltiae (DD-136 strain) 
generally move toward the surface of the substrate and wave their anterior ends in the air. They also have been reported to "leap" as far as $10 \mathrm{~mm}$ (Reed \& Wallace 1965). Heterorhabditis heliothidis juveniles lack these behaviors and tend to move downwards. The moist inner region of the medium where 2ndinstar fly larvae were concentrated may have been either too oxygen-poor or toxic to juveniles of this species. Juvenile $S$. feltiae congregated on the surface of the medium and probably entered 3rd-instar larvae as they moved to the surface to pupate. Examination of the containers 3 days following treatment with nematodes revealed many $S$. feltiae juveniles at the surface of the medium, while no $H$. heliothidis could be found in any of the containers. In poultry manure almost no mortality was observed among 2nd-instar larvae at a host density of 20 larvae per cup, and 3rd-instar mortality was very low $(<30 \%$ at all dosages). Inspection of the manure following nematode introduction indicated that all $H$. heliothidis juveniles were dead within $\mathrm{l} \mathrm{h}$ of application. Nematode mortality was also high among $S$. feltiae larvae, although some parasites could still be seen on the manure surface 5 days posttreatment. Increasing host larval densities from 20 larvae to initial levels of 50 eggs per container had no effect on mortality of 2 nd-instar larvae. Mortality of 3rd-instar larvae was higher, however, and was probably due to the increased probability of the nematodes making contact with host larvae in the initial minutes following application.

Susceptibility of adult flies to nematodes. Adult house flies were as susceptible as larvae to $S$. feltiae and $H$. heliothidis when confined in petri dishes with nematode-treated filter paper. Adult flies were also susceptible to $S$. glaseri, which had no effect on 3rdinstar larvae. Beavers \& Calkins (1984) found that adult and larval $A$. suspensa were equally susceptible to $S$. feltiae and $H$. heliothidis, and that adults were more susceptible than larvae to $S$. glaseri. Laumond et al. (1979) reported that house fly adults were susceptible to $S$. feltiae, although no details were given.

Infection rates were lower when flies were presented with sucrose-baited nematode suspensions, although mortality exceeded $50 \%$ with $S$. feltiae on balls of cotton at the lowest dose of 1,000 nematodes per milliliter of suspension. The large differences observed for $S$. feltiae on the 2 absorbant substrates (cotton balls and cellulose sponges) suggest that nematode bait stations could be effective for flies if an appropriate delivery system were devised.

In summary, our results indicate that while infective-stage juveniles of $S$. feltiae and $H$. heliothidis have a high innate infectivity towards larvae of the house fly, their poor survival and/or searching ability in poultry manure under fly breeding conditions appear to make them poor candidates for biocontrol of filth flies in this habitat. Manure quality (especially moisture content) may influence nematode survival, however, and this warrants further investigation. In another study examining the potential of these nematodes for control of the lesser mealworm, Alphitobius diaperinus (Panzer), we have found that nematode survival and infectivity is considerably greater in the drier medium and manure (ca. $60 \%$ moisture) favored by these beetles in poultry houses than in the moister material $(70-75 \%)$ that we used in the present study with fly larvae (Geden et al., 1985). The nematodes may be more effective in other types of manure (cattle and horse) where fly breeding occurs. Finally, since infectivity can vary as much among conspecific strains as between different species of nematodes (Molyneux et al. 1983), evaluations of additional nematode strains may reveal differences in manure tolerance.

Acknowledgments. The authors wish to thank J.D. Cranford for maintenance of nematode cultures and T.J. Lysyk for assistance in data analysis.

\section{LITERATURE CITED}

Abbott, W.S. 1925. A method of computing the effectiveness of an insecticide. J. Econ. Entomol. 18: 265-67.

Axtell, R.C. 1970. Integrated fly control program for caged poultry houses. J. Econ. Entomol. 63: 400-05.

1981. Use of predators and parasites in filth fly IPM programs in poultry housing. p. 26-43. In: R.S. Patterson, P.G. Koehler, P.B. Morgan \& R.L. Harris, eds., Status of biological control of filth flies. USDA, Agric. Res. SEA A106.2: F64.

Beavers, J.B. \& C.O. Calkins. 1984. Susceptibility of Anastrepha suspensa (Diptera: Tephritidae) to steinernematid and heterorhabditid nematodes in laboratory studies. Environ. Entomol. 13: 137-39.

Bedding, R.A. \& A.S. Molyneux. 1982. Penetration of insect cuticle by infective juveniles of Heterorhabditis spp. (Heterorhabditidae: Nematoda). Nematologica 28: 354-59.

Bedding, R.A., A.S. Molyneux \& R.J. Akhurst. 1983. Heterorhabditis spp., Neoaplectana spp. and Steinernema kraussei: Interspecific and intraspecific differences in infectivity for insects. Exp. Parasitol. 55: 249-57.

Cheng, H.H. \& G.E. Bucher. 1972. Field comparison of the neoaplectanid nematode DD-136 with diazinon for control of Hylemya spp. on tobacco. J. Econ. Entomol. 65: 1761-63.

Dutky, S.R., J.V. Thompson \& G.E. Cantwell. 1964. A technique for the mass propagation of the DD-136 nematode. J. Insect Pathol. 6: 417-22.

Dutky, S.R., J.V. Thompson \& W.S. Hough. 1956. A promising 
new nematode and the associated pathogen for controlling insect pests. Entomol. Res. Branch Circ., Bethesda, Maryland. $5 \mathrm{p}$.

Geden, C.J., R.C. Axtell \& W.M. Brooks. 1985. Susceptibility of the lesser mealworm, Alphitobius diaperinus (Coleoptera: Tenebrionidae) to the entomogenous nematodes Steinernema feltiae, $S$. glaseri (Steinernematidae) and Heterorhabditis heliothidis (Heterorhabditidae). J. Entomol. Sci. 20: 331-39.

Kaya, H.K. 1984. Effect of the entomogenous nematode Neoaplecatana carpocapsae on the tachinid parasite Compsilura concinnata (Diptera: Tachinidae). J. Nematol. 15: 9-13.

Khan, A., W.M. Brooks \& H. Hirschmann. 1976. Chromonema heliothidis n. gen., n. sp. (Steinernematidae, Nematoda), a parasite of Heliothis zea (Noctuidae, Lepidoptera), and other insects. J. Nematol. 8: 159-68.

Lam, A.B.Q. \& J.M. Webster. 1972. Effect of the DD-136 nematode and of a B-exotoxin preparation of Bacillus thuringiensis on leatherjackets, Tipula paludosa larvae. J. Invertebr. Pathol. 20: 141-49.

Laumond, C., H. Mauleon \& A. Kermarrec. 1979. Donnes nouvelles sur le spectre d'hotes et le parasitisme du nematode entomophage Neoaplectana carpocapsae. Entomophaga 24: 13-27.

Molyneux, A.S., R.A. Bedding \& R.J. Ackhurst. 1983. Susceptibility of larvae of the sheep blow fly Lucilia cuprina to various Heterorhabditis spp., Neoaplectana spp. and an undescribed steinernematid (Nematoda). J. Invertebr. Pathol. 42: $1-7$.

Patterson, R.S., P.G. Koehler, P.B. Morgan \& R.L. Harris, eds. 1981. Status of biological control of filth fies. USDA, Agric. Res., SEA A106.2: F64. 212 p.

Poinar, G. O., Jr. 1975. Entomogenous nematodes-A manual and host list of insect-nematode associations. E.J. Brill, Leiden. $317 \mathrm{p}$.
1979. Nematodes for biological control of insects. CRC Press, Boca Raton, Fla. 277 p.

Poinar, G.O., Jr, L. van der Geest, W. Helle \& H. Wassink. 1977. Experiments with organisms from hosts other than Glossina. p. 88-92. In: M. Laird, ed., Tsetse. Int. Dev. Res. Ctr., Can. Res. Dev. Inst. Publ. No. IDRC-077E, Ottawa.

Poinar, G.O., Jr, G.M. Thomas, S.B. Presser \& J.L. Hardy. 1982. Inoculation of entomogenous nematodes, Neoaplectana and Heterorhabditis, and their associated bacteria Xenorhabdus spp. into chicks and mice. Environ. Entomol. 11: 137-38.

Ray, A.A., ed. 1982. SAS user's guide: Statistics. SAS Institute Inc., Raleigh. 584 p.

Reed, E.M. \& H.R. Wallace. 1965. Leaping locomotion by an insect-parasitic nematode. Nature 206: 210.

Regniere, J. \& W.M. Brooks. 1978. Entomogenous microorganisms associated with the Japanese beetle, Popillia japonica, in eastern North Carolina. J. Invertebr. Pathol. 32: 22628.

Webster, J.M. 1973. Manipulation of environment to facilitate use of nematodes in biocontrol of insects. Exp. Parasitol. 33: $197-205$.

Welch, H.E. \& L.J. Briand. 1961. Field experiment on the use of a nematode for the control of vegetable crop insects. Proc. Entomol. Soc. Ont. 91: 197-202.

Welch, H.E. \& J.F. Bronskill. 1962. Parasitism of mosquito larvae by the nematode, DD-136 (Nematoda: Neoaplectanidae). Can. J. Zool. 40: 1263-68.

Wouts, W.M., Z. Mracek, S. Gerdin \& R.A. Bedding. 1982. Neoaplectana Steiner, 1929 a junior synonym of Steinernema Travassos, 1927 (Nematoda: Rhabditida). Syst. Parasitol. 4: 147-54.

\section{ADDENDUM}

After submission of this paper, a preliminary report (Renn, Barson \& Richardson, 1985, Preliminary laboratory tests with two species of entomophilic nematodes for control of Musca domestica in intensive animal units, Ann. Appl. Biol. 106: 229$33)$, presenting data on 2 of the species of nematodes, was published. The data and conclusions on those species are similar to ours and agree with our findings that the nematodes infect larvae and adults of the house fly but do not survive long in chicken manure. 